\title{
LA INTERDISCIPLINARIDAD: POR UN MATRIMONIO ABIERTO DE LA RAZÓN, DE LA MANO Y DEL CORAZÓN
}

\section{Yves Lenoir, Abdelkrim Hasni $(*)$}

SÍNTESIS: El propósito de este artículo es hacer resaltar la existencia de tres conceptos distintos de la interdisciplinaridad y de su uso en el campo de la educación. El primer concepto, europeo francófono, fijado en dimensiones epistemológicas de los saberes disciplinarios y en la racionalidad científica, puede ser calificado de lógico racional, centrado en la búsqueda de significado. El segundo, norteamericano anglófono, es de tipo metodológico y remite a una preocupación de primera instancia por el «saber hacer»; se trata de la lógica instrumental, orientada hacia la búsqueda de la funcionalidad. En cuanto al tercer concepto, que se observa sobre todo en Brasil, se inscribe en la perspectiva fenomenológica, privilegia las dimensiones humanas y afectivas, y expresa una lógica subjetiva dirigida a la búsqueda del sí. Veremos cómo se relacionan estos tres conceptos y cómo pueden utilizarse en el proceso de enseñanzaaprendizaje.

SÍNTESE: O propósito deste artigo é ressaltar a existência de três conceitos diferentes da interdisciplinaridade e de seu uso no campo da educação. O primeiro conceito, europeu francófono, fixado em dimensões epistemológicas dos saberes disciplinares e na racionalidade científica, pode ser qualificado de lógico racional, centrado na busca de significado. O segundo, norte-americano anglófono, é do tipo metodológico e referese a uma preocupação de primeira instância pelo «saber fazer», trata-se da lógica instrumental, orientada para a busca da funcionalidade. Quanto ao terceiro conceito, observado principalmente no Brasil, inscreve-se na perspectiva fenomenológica, privilegia as dimensões humanas e afetivas, e expressa uma lógica subjetiva dirigida à busca do sim. Veremos como se relacionam estes três conceitos e como podem ser utilizados no processo de ensino-aprendizagem.

(*) Director y co-director, respectivamente, del Centro de Investigación en Intervención Educativa [Centre de Recherche surl'Intervention Éducative (CRIE)-( CRIFPE)], Facultad de Educación, Universidad de Sherbrooke, Canadá. 


\section{INTRODUCCIÓN}

El presente artículo tiene como objetivo hacer resaltar la existencia de tres lógicas distintas que animan el recurso a la interdisciplinaridad en educación, y, particularmente, en la enseñanza y en la formación docente. Estas diferentes lecturas del concepto provienen fundamentalmente del hecho de que los actores sociales, los investigadores, los formadores universitarios o los docentes, están involucrados con culturas que son el origen de estas diferenciaciones conceptuales y de su uso. En cada cultura existe un nexo con su mundo específico, que establece un vínculo con el saber propio de ella. ¿O sería el opuesto? ¡Un vínculo con el saber específico que connota un nexo con el mundo singular! De manera más concreta aún, estas dos relaciones se encuentran en estrecha interacción, e influyen en los modos de pensamiento y de acción de una sociedad.

La palabra interdisciplinaridad ha atravesado las fronteras y ha dado la vuelta al planeta. Se utiliza tanto en la francofonía como en los países germano-escandinavos, en los países anglosajones y en los de lengua española y portuguesa. De Nueva Zelanda a B rasil, de Portugal a Noruega, de Chile a Canadá, la palabra es hoy en día de uso común. Podríamos pensar a primera vista, y sin duda de forma un poco ingenua, que la palabra está cargada de un significado socialmente compartido por la totalidad de sus usuarios, y que se caracteriza por algunas perspectivas comunes en el plano de la investigación en educación, así como en el de la formación. Ahora bien, tal aprensión corre el riesgo de dar lugar a malas interpretaciones. En efecto, presentamos la existencia de tres concepciones profundamente diferentes, pero que, pensándolo bien, no sólo aparecen como complementarias sino como indispensables las unas de las otras. Por lo tanto, apelamos a una metáfora inspirada en el ser humano para caracterizar estas tres lógicas: la razón que analiza y que reflexiona, la mano que actúa y que realiza, y el corazón que expresa el cariño y los sentimientos.

Nuestro propósito no se encuentra en hacer un análisis sociohistórico de la evolución de la noción de interdisciplinaridad en el ámbito de la educación. Numerosas publicaciones han intentado indagar acerca de este tema a niveles científicos y educacionales con una perspectiva sociohistórica. Podemos señalar diferentes trabajos, como los de Bourguignon (1997), Ciccorio (1970), Fazenda (1994, 1995b), Flexner (1979), Frank (1988), Gusdorf (1968, 1983), Horn y Ritter (1986), Klein (1990), Knudsen (1937), Kockelmans (1979), Lenoir 
(1995, 1999), Lenoir y Geoffroy (2000), Lenoir y Laforest (2003), Resweber (1981), Stills (1986), Swoboda (1979). Algunos de ellos tratan el asunto de manera global; otros lo analizan desde un punto de vista más local, o en función de un área de aplicación particular. No hay que olvidar los artículos de Fourez (1998) y de Klein (1998), que brindan un enfoque interesante, así como los trabajos de Stichweh (1991), que demuestran que la interdisciplinaridad ya estaba presente en los orígenes de la formación del sistema de las disciplinas científicas en Ios siglos XVIII y XIX.

\section{OBSERVACIONES PRELIMINARES}

Se imponen fundamentalmente dos. En primer lugar, conviene recordar que debemos establecer la distinción entre el tema de la interdisciplinaridad y la cuestión de la unidad del saber, con el fin de eludir toda confusión conceptual y toda interpretación errónea de significados. Es importante evitar caer en la creencia de que se trata de un tema «tan antiguo como el mundo» en lo que se refiere a educación, tal como sugiere, por ejemplo, Choudhuri (1992, p. 199). Si la interdisciplinaridad es una noción reciente, muy ligada al desarrollo del sistema de las disciplinas científicas (Hamel, 1997; Stichweh, 1991), la inquietud sobre la unidad del saber proviene de una preocupación transhistórica (Gusdorf, 1983; Klein, 1990; Lenoir, 1995, 1999; Resweber, 1981; Sinacoeur, 1983). La idea remite ante todo a la unidad del ser humano, a la unidad de su universo cultural, y al significado de la vida (Babossov, 1978; Morin y Piattelli-Palmarini, 1974, 1983), e interviene aún más en términos del horizonte mítico que proyecta una visión idílica de un mundo anticuado, armonioso y homogéneo (Lenoir, 1995).

En segundo lugar, creemos que no estaría bien hablar de interdisciplinaridad sin mencionar la noción de disciplinaridad, estando ésta estructuralmente incluida en la primera. Siguiendo la opinión de varios autores (Cros, 1987; Fourez, 1994; Newell, 1990; Petrie, 1992), así como la de Germain (1991), aunque sin querer caer en la obviedad pero conscientes de que hay que mencionarlo, conviene recordar que la reflexión sobre la interdisciplinaridad sólo tiene sentido en un contexto disciplinario - teniendo a la contextualización histórica como testimonio-, y que «la interdisciplinaridad presupone, por lo menos, la existencia de dos disciplinas de referencia y [la] presencia de una acción recíproca» (Ibíd., p. 143). Sin duda esta evocación no es inútil, puesto 
que existen concepciones Ilamadas «interdisciplinarias», y en varias ocasiones «transdisciplinarias», basadas en una denegación de toda referencia disciplinaria (Lenoiry Sauvé, 1998b). Ahora bien, el concepto de interdisciplinaridad está ligado, histórica y epistemológicamente, al de disciplinaridad. Sin embargo, en el campo dela educación, se trata de una disciplinaridad escolar cuyo significado y cuyos elementos constitutivos difieren de la disciplinaridad científica (Chervel, 1988; Develay, 1992; Hasni, 2001; Messer-Davidow, Shumwayy Sylvan, 1993; Sachot, 1993).

\section{LAS TRES LÓGICAS}

Tal como hemos indicado (Lenoir, 1999, 2001; Lenoiry Sauvé, 1998a), si de una y otra parte del Atlántico se habla del concepto de interdisciplinaridad, las perspectivas no dejan de ser distintas. Esta diferenciación se debe al hecho de que su conceptualización se fundamenta en finalidades diferentes, a distintos objetos de estudio, y a que se recurre a un sistema referencial y a modalidades de aplicación también disímiles (Lenoir y Sauvé, 1998a).

\section{A MANERA DE ILUSTRACIÓN INTRODUCTIVA}

Hace cerca de cuatro años, en una asamblea departamental de la Facultad de Educación de la Universidad de Sherbrooke, en la que se anunciaba que el Ministerio de Educación de Quebec iba a introducir una nueva modificación en la formación para la enseñanza primaria, ycuando ni siquiera los efectos de la reforma anterior (cuya puesta en marcha había tenido lugar en 1994) habían sido evaluados, la reacción automática de algunos colegas fue la siguiente: «¿Cómo la vamos a hacer?». Nunca se ha tratado de poner en tela de juicio la pertinencia o el contenido de esta nueva reforma, la cual iba a conducir otra vez a modificar de manera radical un currículo de formación para la enseñanza preescolar y primaria todavía en proceso de implantación.

La reacción de preocupación de estos colegas nos llamó mucho la atención por su contenido, centrado en las modalidades operacionales, y nos había llevado a imaginar cuál podría ser la respuesta de los universitarios franceses y brasileños frente a una situación similar. Con 
base en nuestra larga experiencia con colegas de estos dos países, nos imaginamos que un universitario francés habría reaccionado de forma distinta. Podría, por ejemplo, haber dicho: «¿por qué una nueva reforma? ¿Por qué es conveniente?», temiendo más por cuestiones relacionadas con los motivos y con el aspecto racional subyacente. En cuanto al colega brasileño, su reacción podría haber sido algo así como: «¿me voy a sentir bien en esta nueva reforma?, ¿me sentiré a gusto?», remitiéndose a procesos introspectivos y a una inquietud por la dimensión humana.

En las siguientes líneas ilustraremos con un ejemplo tres representaciones diferentes de una misma problemática. Se encuentran en la lectura y en el uso que los investigadores de Francia, Quebec y B rasil hacen de la interdisciplinaridad en la educación, en la enseñanza y en la formación docente.

Hay que especificar que, tal como hemos demostrado [Lenoir 1999, 2002 (en prensa); Lenoir y Geoffroy, 2000], Quebec se inscribe del todo en este plano de la corriente norteamericana de habla inglesa. Por tal motivo, desarrollaremos la siguiente argumentación a partir de la realidad estadounidense. No obstante, dos de las tres concepciones de la interdisciplinaridad consideradas aquí parecen predominar, tal como resaltaron Hermerén (1985), Klein (1985) y Lynton (1985) en un seminario de la Organización para la Cooperación y el Desarrollo Económico ( OCDE) que tuvo lugar en la Universidad sueca de Linköping, y otros autores tales como Fourez (1992). Apostel y Vanlandschoot (1994), hablando de los debates que habían tenido lugar a principios de los años 70 en el marco de los trabajos conducidos por la OCDE sobre la interdisciplinaridad, abundan en el mismo sentido. Estos autores destacan la existencia de dos visiones continentales: la primera, muy marcada a nivel social, epistemológica e ideológica, es más europea; la segunda, de orden pragmático, es más bien anglosajona.

\section{LA INTERDISCIPLINARIDAD EUROPEA FRANCÓFONA Y LA BÚSQUEDA DEL SIGNIFICADO, 0 LA LÓGICA RACIONAL}

En la Europa francófona, en particular en Francia, la cuestión interdisciplinaria se considera sobre todo según la tradición elaborada poco a poco a partir del Renacimiento. El desarrollo del pensamiento racional cartesiano, la acción de los filósofos (Voltaire entre otros) y la de los Enciclopedistas del Siglo de las Luces, Ilevaron a una parte de la 
población culta - que constituyó la punta de lanza de la Revolución- a pronunciarse progresivamente contra el oscurantismo, la ignorancia y la opresión social, que, a sus ojos, estaban caracterizados por la Iglesia católica, la realeza y la aristocracia. En consecuencia, en el pensamiento republicano la emancipación de los seres humanos debía pasar por la instrucción. Instruir es, como bien definió Condorcet en 1791, «formar primero a la razón, aleccionar para que sólo se la pueda escuchar a ella, y defenderse del entusiasmo que podría extraviarla u obscurecerla [... ] este es el camino que prescribe el interés de la humanidad, y el principio bajo el cual la instrucción pública debe combinarse» (Condorcet, 1989, p. 185, traducción libre). Ahora bien, la instrucción es liberadora y no puede concebirse sin apelar a la razón, sin una diligencia racional.

En pocas palabras, según la posición republicana francesa, la relación con el saber, con la disciplina científica, resulta ser primordial, puesto que llega a hacerse fiadora de los fundamentos y de los valores de la República, así como de la tradición cultural que ésta ha instaurado. Desde entonces importa poner en tela de juicio el saber disciplinario, cuestionar su significado antes de actuar, y discutir los contenidos cognitivos que hay que someter a los aprendizajes de los futuros ciudadanos adultos. Las perspectivas epistemológicas y didácticas sirven para asegurar estas funciones de análisis del significado, de la pertinencia, de la calidad de los objetos de enseñanza propuestos en el sistema escolar, así como de su adaptación y adecuación a las capacidades de los alumnos. Ante todo, se hace hincapié en saber enseñar (el polo objeto), y se prioriza la transmisión valorada de las disciplinas escolares. Esta concentración en el «polo objeto» se explica por el renacimiento de una construcción social inherente al ser instruido, hecha para enseñar a los futuros ciudadanos de la República.

Para buscar una síntesis conceptual, la interdisciplinaridad (de tipo académico, dado que constituye fundamentalmente una apuesta social y epistemológica universitaria) tiene un carácter reflexivo y crítico, o está orientada hacia un trabajo de unificación del saber científico (en el sentido de una estructuración jerárquica de las disciplinas o en el de una superciencia, una metateoría o una metadisciplina), o está dirigida hacia un trabajo de reflexión epistemológico sobre los saberes disciplinarios que interactúan. En el plano escolar, el debate se refiere de manera prioritaria a la pertinencia de las conexiones entre los saberes disciplinarios que son el objeto de la enseñanza. En coherencia con la lógica racional socialmente privilegiada, la cuestión del significado se encuen- 
tra en el corazón de la perspectiva epistemológica (o filosófica en ciertos casos), y se refiere al sistema de las disciplinas científicas. Así mismo, la interdisciplinaridad es abordada en función de las interacciones internas a sus disciplinas.

\section{LA INTERDISCIPLINARIDAD NORTEAMERICANA ANGLOSAJONA Y LA BÚSQUEDA DE LA FUNCIONALIDAD, O LA LÓGICA INSTRUMENTAL}

En Estados Unidos el recurso a la interdisciplinaridad se emprende, en primer lugar, con una perspectiva del todo distinta, es decir, la instrumental. Desde el punto de vista sociohistórico, la opción norteamericana es el resultado de un conjunto de factores que, poco a poco, han conducido al rechazo del modelo europeo, tras su independencia de la Gran Bretaña (Lenoir, 2002).

En la concepción norteamericana lo que lleva al ser humano a la libertad no está relacionado directamente con los conocimientos, sino con la capacidad de actuar en y sobre el mundo, es decir, que educar equivale a instrumentar en un doble sentido: el de la práctica y el de las relaciones humanas y sociales. Así es como se desarrolla, en la segunda mitad del siglo XIX, una concepción «vocacionalista», centrada, por una parte, en el desarrollo de símbolos destinados a conciliar la ética protestante con el nuevo orden industrial en auge, $y$, por otra, en el desarrollo de formaciones profesionales (Geoffroyy Lenoir, 2000; Geoffroy, Lenoiry Hasni, 1999). Los trabajos de Kliebard (1986, 1992a, 1992b), de Rudolph (1977), y de Tanner y Tanner (1990) hacen ver que esta concepción resulta de una preocupación nuclear por la inserción y la integración del ser humano en una sociedad joven, multiétnica, y de diversas culturas y creencias religiosas. Es decir, que lo que importa ante todo es asegurar la producción de los seres humanos, de las personas individuales capaces de participar de manera armoniosa en las actividades sociales, políticas y económicas de una comunidad que tiene su base en la diversidad. Además, esta concepción es consecuencia -al menos al principio- del rechazo de la violencia que caracterizó la creación y la institucionalización de los Estados-naciones europeos entre comienzos del siglo XVII y mediados del XX.

Kliebard (1992a) explica de qué forma el principio del humanismo, que pasa de una concepción mentalista y culturalista propia de la concepción británica de la educación a la racionalidad instrumental, 
ha sido reemplazado por el de profesionalismo (vocationalism) en la política curricular de Estados Unidos. Esta teoría está muy relacionada con el origen, con el paradigma dominante del taylorismo -la eficacia social (social efficiency) - y con su aplicación en el campo de la educación por la Tyler Rationale, que llega a ser la principal guía de las políticas y de las prácticas curriculares adoptadas en el país. Desde esta perspectiva, Withehead (1929) insiste en que «la cultura general y la ciencia salgan al encuentro de la acción y participen en el progreso de la sociedad». Lejos de considerar la cultura y la ciencia como fines en sí mismos, no tiene objeción en que la educación, con independencia de su forma y de su contenido, sea útil: «Los pedantes desprecian una educación útil. ¿Pero, qué puede ser la educación si no es útil? ¿Se trata de un talento que debe conservarse en secreto en un portafolios? Por supuesto, la educación debe ser útil, sin importar la meta que se tenga en la vida. Así fue tanto para San Agustín como para Napoleón. Y eso porque la inteligencia de la vida es útil» [Drèze y Debelle, 1968, p. 67 (traducción libre); Lessard y Tardif (por publicar)]. Así mismo, se advierte un fuerte nexo en el plano de las finalidades del sistema escolar, entre una visión pragmática, instrumental (el «saber hacer»), y una preocupación por la inserción social, por la adhesión a normas y a valores que caracterizan al pueblo americano (el «saber ser»).

En Estados Unidos uno no se pregunta acerca del problema del saber, puesto que no es éste el que hace libre -a los ojos de los pensadores que han elaborado el discurso ideológico de legitimación del orden y del funcionamiento social-, tanto más cuanto que el discurso ideológico creador de la sociedad norteamericana está basado en la idea de «destino manifiesto». En efecto, ese país se fundamenta en la convicción de que procede de un «destino manifiesto», el de ser el «nuevo pueblo del srael», con la llegada de los puritanos calvinistas a esa «nuevaJ erusalén» que era la América del siglo XVII, de ser los «padres fundadores» de un nuevo «pueblo elegido» con una misión civilizadora: se trata de trabajar por la felicidad de la humanidad, ofreciéndole el ejemplo norteamericano en cuanto a virtud, a libertad y a progreso. En el corazón de este concepto se encuentra la creencia en la superioridad norteamericana. La idea de «destino manifiesto» y de superioridad se vuelve a encontrar en el siglo XIX para justificar el expansionismo norteamericano hacia el interior [fase de continentalización (1803-1848)] y hacia el exterior [fase imperialista (1866-1904)], el genocidio de los amerindios que no son protegidos por la Providencia (mueren por las epidemias, los blancos, no), y en que obstaculizan el efecto del Evangelio (es decir, la Conquista del Oeste). La 
justificación ideológica (la superioridad anglosajona) y teológica (la Providencia), reforzadas por los mitos de la Conquista del Oeste y del melting pot, son fuentes de cohesión social, pero tal cohesión se define en un marco muy preciso ( WASP $=$ White-Anglo-Saxon-Protestant), que procede a la exclusión de la gente de color, de los amerindios y de los hispanos mexicanos. No son ciudadanos, sino, en el mejor de los casos, residentes (Marienstras, 1988). El sigloxIX es testigo del establecimiento de un proceso de secularización del mito religioso (reemplazado por la idea de democracia), pero este mito sigue estando muy vivo, hasta el punto de que Madeleine Albright declaró en pleno 1977: «Nosotros, los americanos, tenemos una ventaja sobre las demás naciones. Sabemos quiénes somos y en qué creemos. Somos unos constructores. Nuestra responsabilidad no es la de actuar como prisioneros de la historia, sino de hacer la historia [... ] Tenemos una razón de ser [... ] y la fe, que, si somos fieles a nuestros principios, nos hará triunfar.» (traducción libre).

En resumen, lo que hace libre es la capacidad de actuar de manera autónoma en y sobre el mundo. La cuestión principal no es la del saber ni la del significado, puesto que el significado lo proporciona la sociedad norteamericana, considerada a priori como buena y justa dado que depende de un «destino manifiesto» querido por Dios, sino de la funcionalidad, la del «saber hacer», que requiere un cierto «saber ser», es decir, que la relación con el sujeto es primordial.

En tal contexto, la interdisciplinaridad se basa sobre todo en interacciones sociales externas, pues está pensada en términos de búsqueda de respuestas operacionales a preguntas hechas dentro de la sociedad. Centrada en la resolución de problemas sociales, se puede hablar, entonces, de una interdisciplinaridad de proyectos en los que el saber requerido es útil y operacional de inmediato. En el plano escolar, se trata más bien de modalidades de ajuste de situaciones de aprendizaje a partir de modelos organizacionales muy numerosos (Lenoir y Sauvé, 1998b) para favorecer la prosecución de finalidades de integración social, así como de la parte instrumental de la realidad. De igual modo, a la búsqueda de significado se opone la búsqueda de funcionalidad.

1 Ver Marienstras $(1976,1988)$ así como, por ejemplo, el número especial de la revista L'Histoire (2000) sobre «El imperio americano». 
Siguiendo la lógica norteamericana, surge con nitidez la idea de que la interdisciplinaridad no es la finalidad del proceso de enseñanzaaprendizaje, sino sólo un medio que se considera eficaz. La integración sí tiene esta finalidad, que debe ser comprendida bajo una doble perspectiva, pues favorece una relación educativa integradora, una integrative education (Klein, 1990). Por una parte, desde el punto de vista de la docencia, requiere del formador docente un enfoque integrador (integrative approach), que sirve como base y sostén del recurso a la interdisciplinaridad. Por otra, desde la perspectiva de los aprendizajes, la integración es un proceso global (la integración de los procesos de aprendizaje, de los integrative processes) que requiere recurrir a gestiones de aprendizaje. Éstas intervienen como procesos mediadores en la relación de objetivación que se establece entre un sujeto (alumno) y los objetos de aprendizaje, siendo estos mismos procesos mediadores objetos de aprendizaje. Pero la integración también es, siempre desde el punto de vista de los aprendizajes, el producto de dichos procesos (la integración de los saberes, el integrated knowledge). Estas dos dimensiones del proceso de aprendizaje -los procesos mediadores integradores que abarcan el uso de gestiones de aprendizaje, y el producto de estos procesos, es decir, el saber adquirido- son indisociables y necesarias. En consecuencia, la integración es considerada como un proceso interno y transportable de construcción de productos cognitivos, que pertenece al sujeto y que requiere el apoyo apropiado de una tercera persona para actuar como mediadora momentánea (el docente) y para establecer condiciones de aprendizaje favorables con orientaciones integradoras.

Esa es la razón por la cual, siguiendo la lógica que prevalece en Estados Unidos, donde la relación con el sujeto es primordial, importa preguntarse acerca de las perspectivas pedagógicas y organizacionales -el «cómo hacer»- que mejor favorezcan el establecimiento de dispositivos apropiados para permitir a los sujetos (alumnos) alcanzar estas finalidades, facilitándoles, por una parte, integrar, a través de sus aprendizajes, las normas y los val ores sociales contenidos en el currículo, $y$, por otra, desarrollar las habilidades instrumentales requeridas para actuar en y sobre el mundo. En ese sentido, en Estados Unidos la interdisciplinaridad sería la respuesta a la problemática del «saber hacer» y la integración del «saber ser». 


\section{LA INTERDISCIPLINARIDAD LATINOAMERICANA BRASILEÑA O LA LÓGICA SUBJETIVA}

A estos dos enfoques de la interdisciplinaridad - uno formado a partir del saber (el «polo objeto») y otro a partir del «saber hacer» y de un cierto «saber ser» (el «polo sujeto»), conviene agregar un tercero, que encontramos en buena parte en la concepción brasileña de la interdisciplinaridad. En efecto, el recurso a la interdisciplinaridad se concibe, por lo general, de forma distinta en Brasil. Fazenda (1991, 1994, 1995a, 1995b, 2001a, 2001b), una investigadora nativa de prestigio por sus trabajos sobre la interdisciplinaridad, junto con sus colaboradores, con profesores y con estudiantes, actúan de forma explicita como testigos de esta nueva orientación.

La perspectiva adoptada es de tipo fenomenológico, y, en ese sentido, recurre a la interacción simbólica. Pone en evidencia al actor -en especial al docente como vector interdisciplinario-, y está orientada a la subjetividad de los agentes como partes de lo cotidiano, y hacia su intersubjetividad en el plano metodológico. El hecho social está constituido sobre todo por intersubjetividades (Schütz, 1987), que provienen de las interacciones que producen entre ellas los actores sociales. En tal caso, el proceso de construcción continuo del individuo en situación constituye la preocupación principal, y se explica no sólo por el conocimiento de sí mismo sino también del otro (Fazenda, 1998). Ahora bien, para do Espírito Santo (1995a), la práctica interdisciplinaria suscita un autoconocimiento. Cuatro principios forman la base de una práctica docente interdisciplinaria: la humildad, la coherencia, la expectativa y la audacia. Algunos atributos son propios de dicha práctica, y determinan o identifican estos principios: se trata de la afectividad, del respeto y del despego, que llevan a los intercambios intersujetivos y a la colaboración (Fazenda, 2001b). En el centro de esta concepción se encuentra el carácter indisociable de la interacción, como fundamento y como motor de la constitución del ser humano. Es por eso por lo que, según la opinión de Fazenda (1998), no se modifica la conducta de los docentes con la imposición de prescripciones, sino a través de un proceso particular de transformación en el cual el docente se convierte en un participante comprometido en un sistema de interacciones con los alumnos. EI descubrimiento y la valorización de lo que son los docentes, de sus maneras de ser y de actuar, constituye el punto de partida del proceso de formación que tiende a proponer alternativas a sus prácticas. 
En la lógica brasileña, muy subjetiva (e intersubjetiva), la interdisciplinaridad no tiene una función reflexiva ni instrumental en el saber. Su finalidad es otra: busca la realización del ser humano promoviendo una concentración en el «yo» integrador, no en las relaciones entre las disciplinas, y mucho menos en el actuar funcional, pragmático. Adhiriéndose a una perspectiva afectiva, la interdisciplinaridad está volcada en las interacciones internas sobre el tema, buscando respuestas a preguntas personales. Se trata de lo que podríamos llamar una interdisciplinaridad introspectiva, basada en la búsqueda del «sí».

Hay que señalar que, si las concepciones norteamericana y brasileña requieren la búsqueda de un «saber ser», éste remite a dos significados. En el caso de los Estados Unidos, el «saber ser» se refiere a la integración social y acompaña al «saber hacer», que es primordial. En Brasil, el «saber ser» resulta más de la introspección, de la preocupación personal, y caracteriza la concepción de la interdisciplinaridad como medio de emancipación. En esa óptica, el recurso a la interdisciplinaridad permite a los seres humanos crecer.

La tabla siguiente muestra una síntesis de las tres concepciones: francesa, norteamericana y brasileña.

\section{CONCLUSIÓN}

Hemos presentado tres visiones de la interdisciplinaridad: la europea de habla francesa, que busca el significado con una perspectiva racional, y que remite al saber homologado del sistema de las disciplinas científicas; la norteamericana anglosajona, que busca la funcionalidad con una perspectiva instrumental recurriendo al «saber hacer» (competencias profesionales), con el afán de satisfacer expectativas sociales; y la visión latinoamericana brasileña, que busca la realización del «yo» por medio de la subjetividad y de la intersubjetividad (un «saber ser» fuertemente connotado de dimensiones humanas, en particular de orden afectivo). Esas visiones se han elaborado y actualizado con base en racionalidades distintas (ideológicas, políticas, económicas, culturales), que entran en el marco del desarrollo sociohistórico de cada uno de esos Estados-naciones que son Francia, Estados Unidos y B rasil.

Estas tres visiones, que a primera vista podrían parecer antitéticas, están lejos de serlo cuando las analizamos con ansias de 


\section{TABLA}

Tres perspectivas de aprensión de la interdisciplinaridad

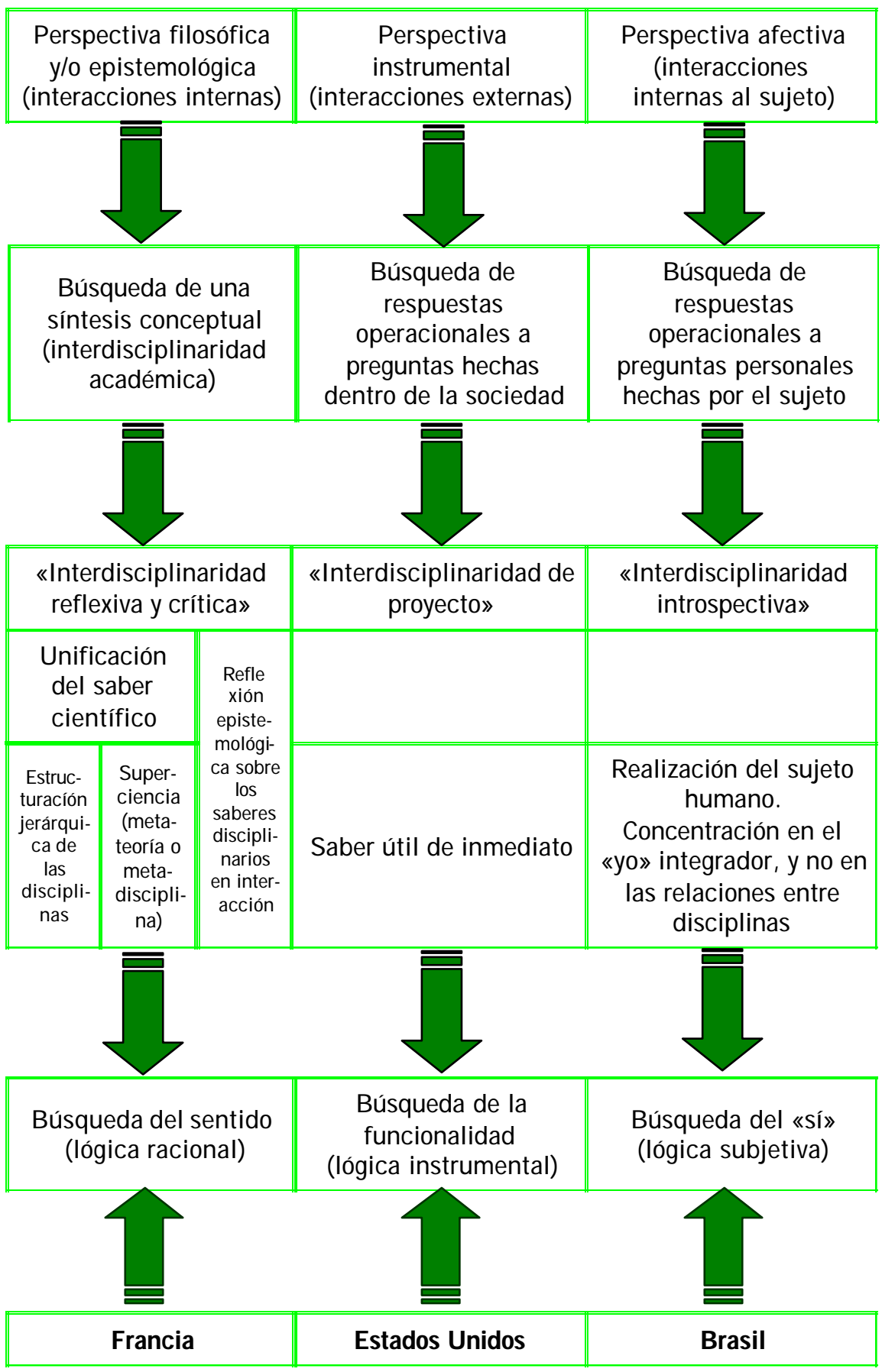


comparación. Al contrario, si las tres han de ser preservadas y mantenidas, hay que recurrir a ellas de forma complementaria. El enfoque equilibrado de un pensamiento y de una práctica interdisciplinaria requieren un maridaje abierto, el de la razón, es decir, el de la mente, el de la reflexión, el del pensamiento crítico, y, metodológicamente, el del trabajo de conceptualización (plantear y construir la problemática); el de la mano, es decir, el de la realización de la acción productiva (resolver la problemática una vez construida); así como el del corazón, es decir, el de la toma en consideración de las dimensiones humanas. No basta con concebir la interdisciplinaridad al rededor de objetos del saber, ni con preguntarse sobre su significado en los planos ontológico, epistemológico y conceptual (el «por qué» y el «qué»), ni tampoco con concebirla al rededor del sujeto y de preguntarse por las modalidades de accesión a los saberes (el «cómo»); de ahí la importancia concedida a los aspectos pedagógicos y organizacionales de un punto de vista curricular, sino también hay que introducir las dimensiones humanas en las relaciones sociales que se establecen en el marco de un proceso de enseñanzaaprendizaje. Negar o rechazar una de estas tres concepciones sería una grave equivocación, si de lo que se trata es de concebir y de actualizar un enfoque interdisciplinario innovador, orientado hacia una formación integradora. Razón, mano y corazón constituyen tres modos indisociables de aprehender el enfoque interdisciplinario.

\section{BIBLIOGRAFÍA}

APOSTEL, L. y VANLANDSCHOOT, J . (1994): «Interdisciplinarity: The Construction of Worldviews and the Dissemination of Scientific Results», en Issues in lintegrative Studies, 12, pp. 9-22.

BABOSSOV, E. M. (1978): «L'homme, sujet d'études interdisciplinaires», en Diogène, 104, pp. 24-38.

BOURGUIGNON, A. (1997) : «Dela pluridisciplinaritéà la transdisciplinarité», en Bulletin du CIRET, pp. 9-10, accesible en la dirección http://perso.club-internet.fr/nicol/ ciret/.

CHERVEL, A. (1988): «L'histoire des disciplines scolaires. Réflexions sur un domaine de recherche», en Histoire de l'éducation, 38, pp. 59-119.

CHOUDHURI, I. N. (1992): «La recherche et la pratique interdisciplinaire dans le domaine de l'alphabétisation», en E. Portella (dir.): Entre savoirs. L'interdisciplinarité en acte: enjeux, obstacles, résultats, pp. 199-210, Toulouse, Érès. 
CICCORIO, E. A. (1970): «Integration in the Curriculum: An Historical and SexanticIrquiry», enMain Currents in Modern Thought, 27, pp. 60-62.

CONDORCET, M. J. A. Caritat, marqués de (1989): Écrits sur l'instruction publique, vol. II: Rapport sur l'instruction (texto presentado, anotado y comentado por Charles Coutel y Catherine Kintzler), París, Édilig (1. ${ }^{a}$ ed. 1791-1792).

CROS, F. (1987): "“L'interdisciplinarité". Perspectives documentaires en sciences de l'éducation», pp. 11, 35-47.

DEVELAY, M. (1992): De l'apprentissage à l'enseignement, París, ESF.

ESPíRITO SANTO, R. C. do (1995a): «A questão do autoconhecimento na prática interdisciplinar», en I. C. A. Fazenda (dir.): A académia vai à escola, Campinas, Papirus Ed. Cortez Ed.

FAZENDA, I. C. A. (dir.) (1991): Práticas interdisciplinares na escola, São Paulo,

- (1994): Interdisciplinaridade: história, teoria e pesquisa, Campinas, PapirusEd.

- (dir.). (1995 a): A academia vai à escola, Campinas, Papirus Ed.

- (1995b): «Critical-historical Review of Interdisciplinary Studies in B razil», en Association for Integrative Studies Newsletter, 17, 1, pp.1-9.

- (1998): «La formation des enseignants pour l'interdisciplinarité: synthèse de recherches effectuées au Brésil», en Revue des Sciences de l'Éducation, XXIV, 1, pp. 95-114.

- (2001a): «Avancées théorico-méthodologiques de la recherche sur l'interdisciplinarité au Brésil», en Y. Lenoir, B. Rey el. Fazenda (dir.): Les fondements del'interdisciplinarité dans la formation à l'enseignement, pp. 147-156, Sherbrooke, Éditions du CRP.

- (dir.) (2001b): Dicionário em construção, São Paulo, Cortez Edit.

FLEXNER, H. (1979): «The Curriculum, the Disciplines, and Interdisciplinarity in Higher Education: Historical Perspective», en J. J. Kockelmans (dir.): Interdisciplinarity and Higher Education, pp. 93-122, Pittsburg, PA, The Pennsylvania State U niversity Press.

FOUREZ, G. (1992): La construction des sciences. Les logiques des inventions scientifiques. Introduction à la philosophie et à l'éthique des sciences (2 . a ed. revisada), Bruselas, De Boeck Université.

- (1994): Alphabétisation scientifique et technique. Essai sur les finalités de I'enseignement des sciences, Bruselas, De Boeck Université.

- (1998): «Se représenter et mettre en œuvre l'interdisciplinarité à l'école», Revue des Sciences de l'Éducation, XXIV(1), pp. 31-50.

FRANK, R. (1988): «"Interdisciplinarity": The First half Century», en Issues in Integrative Studies, 6, pp. 139-151.

GeOFFROY, Y. y LENOIR, Y. (1999): Differing Conceptions of Interdisciplinarity in Education: The American, French and Quebec Perspectives. Texto de la comunicación de la $21^{\text {th }}$ Annual Association for Integrative Studies Conference, Napierville, Illinois, 30 de septiembre-3 de octubre. 
- (2000): Differents Rationales at Work: Conceptions of the Curriculum Conception in Francophone Europe, in USA, in the German and Scandinavian World and in Quebec. Texto de la comunicación en la Canadian Association for Curriculum Studies (CACS), $28 .^{\circ}$ Congreso anual de la Canadian Society for the Study of Education (CSSE), Edmonton, 24-27 de mayo.

GeRmaIN, C. (1991): «Interdisciplinarité et globalité: remarques d'ordre épistémologique», en Revue des Sciences de l'Éducation, XVII, 1, pp. 142-152.

GUSDORF, G. (1968): «Interdisciplinaire (connaissance)», en Encyclopedia Universalis, vol. 8, pp. 1086-1090, París, Encyclopedia Universalis.

- (1983): «Passé, présent, avenir de la recherche interdisciplinaire», en L. Apostel, J .-M. Benoist, T. B. Bottomore, K. E. Boulding, M. Dufrenne, M. Eliade, C. Furtado, G. Gusdorf, D. Krishna, W. J . Mommsen, E. Morin, M. Piatteli-Palmarini, M. A. Sinacœur, S. N. Smirnov y J. Ui: Interdisciplinarité et sciences humaines (vol. 1, pp. 31-51), París, UNESCO.

HAMEL, J . (1997): Précis d'épistémologie de la sociologie, París, L'Harmattan.

HASNI, A. (2001): Les représentations sociales d'unedisciplinescolaire- I'activité scientifique- et de sa place au sein des autres disciplines formant le curriculum chez des instituteurs marocains. Tesis de doctorado en educación, Universidad de Sherbrooke.

Hermerén, G. (1985): «Interdisciplinarity Revisited: Promises and Problems», in L. Levin el. Lind (dir.): Interdisciplinarity Revisited: Re-assessing the Concept in the Light of Institutional Experience, pp. 15-25, Estocolmo, OCDE/CERI, Swedish National B oard of Universities and Colleges, Linköping University.

HORN, T. C. yRITTER, H. (1986): «Interdisciplinary History: A Historiographical Review», en History Teacher, 19 (3), pp. 427-448.

KLEIN, J . T. (1985): «The Interdisciplinary Concept: Past, Present and Future», en L. Levin el. Lind (dir.): Interdisciplinarity Revisited: Re-assessing the Concept in the Light of Institutional Experience, pp. 104-136, Estocolmo, OCDE/CERI, Swedish National Board of Universities and Colleges, Linköping University.

KLEIN, J. T. (1990): Interdisciplinarity: History, Theory, and Practice, Detroit, MI, Wayne State University Press.

- (1998): «L'éducation primaire, secondaire et postsecondaire aux ÉtatsUnis: vers I'unification du discours sur l'interdisciplinarité», en Revue des Sciences de I'Éducation, XXIV, 1, pp. 51-74.

KLIEBARD, H. (1986): The Struggle for the American Curriculum: 1893-1958, Boston, MA, Routledge y Kegan Paul.

- (1992a): Forging the American Curriculum. Essays in Curriculum History and Theory, Nueva York-Londres, Routledge.

- (1992b): «Constructing a History of the American Curriculum», en P. H. J ackson (dir.): Handbook of Research on Curriculum. A Project of the American Educational Research Association, pp. 157-184. Nueva York, Macmillan. 
KNUDSEN, C. W. (1937): «What do Educators mean by “integration“»?, en Harvard Educational Review, VII, 1, pp. 15-26.

KoCKELMANS, J . J . (1979): «Scienceand Discipline. Some Historical and Critical Reflections», en J J. Kockelmans (dir.): Interdisciplinarity and Higher Education, pp. 11 45, Filadelfia, PA, The Pennsylvania State University Press.

LENOIR, Y. (1995): «L'interdisciplinarité: aperçu historique de la genèse d'un concept», en Cahiers de la recherche en éducation, 2, 1, pp. 1-39.

- (1999): «Interdisciplinarité·», in J . Houssaye (dir.), Questions pédagogiques. Encyclopédie historique, pp. 291-314, París, Hachette.

- (2001): «L'interdisciplinarité dans la formation à l'enseignement: des lectures distinctes en fonction de cultures distinctes », en Y. Lenoir, B. Rey e I. Fazenda (dir.): Les fondements de l'interdisciplinarité dans la formation à l'enseignement, pp. 1736, Sherbrooke, Éditions du CRP.

- (2002) : «Les réformes actuelles de la formation àl'enseignement en France et aux États-Unis: éléments de mise en perspective sociohistorique à partir du concept d'éducation», en Revue Suisse des Sciences de l'Éducation, 24, 1, pp. 91-128.

- (por publicar): «Le "Rapport Parent" ou le début de l'ancrage de l'école québécoise dans la logique anglophone nord-américaine, en Revue Canadienne de l'Éducation.

LENOIR, Y. y GeOFFROY, Y. (2000): «Conceptions de l'intégration dans I'enseignement primaire auxÉtats-Unis et au Québec: une perspective sociohistorique», en Carrefours de l'éducation, 10, pp. 118-154.

LENOIR, Y. y LAFOREST, M. (2003): «Préoccupations interdisciplinaires dans I'enseignement primaire québécois: eléments de contextualisation sociohistorique », en Y. Lenoir y M. Sachot (dir.): Didactique et formation interdisciplinaire. La formation didactique des enseignants du primaire: approches disciplinaires ou interdisciplinaires?, pp. 55-93, Bruselas, De Boeck Université.

LENOIR, Y. y SAUVÉ, L. (1998a): «De l'interdisciplinarité scolaire à I'interdisciplinarité dans la formation à l'enseignement: un état de la question. 1 - Nécessité de l'interdisciplinarité et rappel historique», en Revue Française de Pédagogie, n.. 124, pp. 121-153.

- (1998b): «De l'interdisciplinarité scolaire à l'interdisciplinarité dans la formation à l'enseignement: un état de la question. 2 - Interdisciplinarité scolaire et formation interdisciplinaire à l'enseignement». Revue Française de Pédagogie, n. 0125 , pp. 109-146.

LESSARD, C. y TARDIF, M. (por publicar): «La nature et la place d'une formation professionnelle sel on les conceptions de l'université », en Y. Lenoir y M.-H. Bouillier-Oudot (dir.): Savoirs professionnels et curriculum de formation de professionnels. Une variété de situations, une variété de conceptions, une variété de propositions, Québec, Presses de l'Université Laval.

L'HISTOIRE (2000): L'empire américain, 7, pp. 7-106 (número fuera de serie). 
LYNTON, E. A. (1985): «Interdisciplinarity: Rationales and Criteria of Assessment», en L. Levin el. Lind (dir.): Interdisciplinarity Revisited: Re-assessing the Concept in the Light of Institutional Experience, pp. 15-25, Estocolmo, OCDE/CERI, Swedish National Board of Universities and Colleges, Linköping University.

MARIENSTRAS, É. (1976): Les mythes fondateurs de la nation américaine, París, François Maspéro. Gallimard

- (1988): Nous le peuple. Les origines du nationalisme américain, París,

MeSSER-DAVIDOW, E.; S HUMWAY, D. R. y SYLVAN, D. J . (dir.) (1993): Knowledges: Historical and Critical Studies in Disciplinarity, Charlottesville, VA, University Press of Virginia.

MORIN, E. yPIATTELLI-PALMARINI, M. (dir.) (1974):L'unitédel'homme. Invariants biologiques et universaux culturels, París, Seuil.

MORIN, E. y PIATTELLI-PALMARINI, M. (1983): «L'unité de I'homme comme fondement et approche interdisciplinaire», en L. Apostel, J .-M. Benoist, T. B. Bottomore, K. E. B oulding, M. Dufrenne, M. Eliade, C. Furtado, G. Gusdorf, D. Krishna, W. J. Mommsen, E. Morin, M. Piatteli-Palmarini, M. A. Sinacœur, S. N. Smirnov y J. Ui: Interdisciplinarité et sciences humaines, vol. 1, pp. 191-215, París, UNESCO.

NeWELL, W. H. (1990): «Interdisciplinary Curriculum Development», Issues in Integrative Studies, 8, pp. 69-86.

PetRIE, H. G. (1992): «Interdisciplinarity Education: Are we face with Insurmountable Opportunities?», en Review of Research in Education, 18, pp. 299-333.

Resweber, J. P. (1981): La méthode interdisciplinaire, París, Presses Universitaires de France.

RudolPh, F. (1977): Curriculum. A History of the American Undergraduate Course of Study Since 1636, San Francisco, CA, J ossey-Bass.

SACHOT, M. (1993): "La notion de "discipline scolaire": éléments de constitution», en J.-P. Clément y M. Herr (dir.): L'identité de l'éducation physique scolaire au XXe siècle: entre l'école et le sport, pp. 127-147, Clermont-Ferrand, Ed. AFRAPS.

SCHÜTZ, A. (1987): Le chercheur et le quotidien, París, Méridiens Klincksieck.

SINACEUR, M. A. (1983): «Qu'est-ce que l'interdisciplinarité?», en L. Apostel, J .-M. B enoist, T. B. Bottomore, K. E. Boulding, M. Dufrenne, M. Eliade, C. Furtado, G. Gusdorf, D. Krishna, W. J. Mommsen, E. Morin, M. Piatteli-Palmarini, M. A. Sinacœur, S. N. Smirnov y J. Ui: Interdisciplinarité et sciences humaines, tomo I, pp. 21-29, París, UNESCO.

STICHWEH, R. (1991): Études sur la genèse du système scientifique moderne (trad. F. Blaise), Lille, Presses Universitaires de Lille.

STILLS, D. L. (1986): "A note on the Origine of Interdisciplinarity», en ITEM, Social Science Research Council, 40, 1, pp. 17-18.

SWOBODA, W. W. (1979): «Disciplines and Interdisciplinarity: A Historical Perspective», en J . J . Kockelmans (dir.): Interdisciplinarity and Higher Education, pp. 93122, Pittsburg, PA, The Pennsylvania State University Press. 
Y. LENOIR, A. HASNI

TANneR, D. yTANnER, L. (1990): History of the School Curriculum, Nueva YorkLondres, Macmillan/Collier Macmillan.

WhiteheAd, A. N. (1929): The aims of Education, Londres, Williams y Norgate. 


\title{
Contactar
}

Revista lberoamericana de Educación

\author{
Principal OEI
}

